Stephen L. Jury

\title{
Lichens collected during the fifth "Iter Mediterraneum" in Morocco, 8-27 June, 1992
}

\author{
Abstract \\ Jury, S. L.: Lichens collected during the fifth "Iter Mediterraneum" in Morocco, 8-27 June, 1992. \\ - Bocconea 26: 145-149. 2013. - ISSN 1120-4060 (print), 2280-3882 (online). \\ The Lichens collected by S.L. Jury and R. Wilson during Iter Mediterraneum V are listed, to- \\ gether with the indication of the institutions where the material is deposited. \\ Key words: Flora of Morocco, Lichens, Itinera Mediterranea, OPTIMA. \\ Address of the author: Stephen L. Jury, 3 Grove Road, Leighton Buzzard, LU7 1SF, U.K. \\ stephenjury21@btinternet.com
}

Throughout Iter V (8-27th June, 1992), Stephen Jury and Rupert Wilson collected 314 gatherings of bryophytes and 195 of lichens. These were included in one series of S.L. Jury's personal collection numbers (10452 - 10970) which also contained a few seed gatherings without voucher specimens. A number of the cryptogams proved to be identical, and if they came from the same habitat at the same site location, they were amalgamated, while some others were split into two or more as they proved to be mixed collections. These were designated by adding a lower case letter after the number.

Records in the University of Reading Herbarium show that a lot of duplicates were distributed in summer 1992 in order to obtain identifications. They were all sent out in packets with collection details and a spare label included to be returned to Reading with any determination and the name of the identifier. In fact, none of these were returned, though Dr H. Sipman, Berlin, sent a list of determinations he made the same summer. A visit to Reading by the distinguished lichenologist, Professor Per Magnus Jørgensen, enabled further lichen specimens to be determined.

The main set of lichens was repatriated to the herbarium IAV at the Institut Agronomique et Vétérinaire Hassan II, Rabat, whilst other sets were distributed as follows:

SEV 245 (85 lichens [set 4] and 160 bryophytes [set 5]).

PAL 322 (63 lichens [set 5], 257 bryophytes [set 3] and 2 fungi.

B 146 (46 lichens [set 6] and 100 bryophytes [set 7]). 
LIV 250 (30 lichens [set 7] and 220 bryophytes [set 4]).

CAME 155 (30 lichens [set 8] and 125 bryophytes [set 6]).

\section{Lichen identifications}

The Lichens which have been identified at generic or specific level are listed below for each collecting locality. The numbers in square brackets at the end of each location are the numbers of the vascular collecting sites as given in detail in the preceding Checklist of vascular plants.

Many of the determinations are provisional, and any corrections and additions are welcomed.

C. 12 km from Rabat on road to Meknès, Forêt de la Maâmora, 9 June [1]

10454 Cladonia rangiformis Hoffm.

C. $15 \mathrm{~km}$ from El Hajeb on road to Ifrane, Forêt de Jaaba, 9 June [4]

10464 Parmelia tiliacea (Hoffm.) Ach.

10465 Anaptychia ciliaris (L.) Koerb.

10466 Physconia venusta (Ach.) Poelt

10467 Usnea

10469 Evernia prunastri (L.) Ach.

10470 Parmelia carporrhizans (Taylor) Poelt \& Vezda

10472 Pertusaria

10473 Leproloma

10474 Physconia distorta (With.) Laund.

10475 Parmelia tiliacea (Hoffm.) Ach.

High Atlas, c. $15 \mathrm{~km}$ from Midelt to Cirque du Jaffar, Jbel Ayachi, 10 June [7]

10478 Lecanora

10481a Caloplaca

10481b Candelariella

Middle Atlas, $22 \mathrm{~km}$ to Ain Leuh from Azrou-Midelt road, 11 June [10]

10493 Anaptychia ciliaris (L.) Koerb.

10494 Parmelia tiliacea (Hoffm.) Ach.

10495 Physconia perisidiosa (Erichsen) Moberg

10496 Physconia venusta (Ach.) Poelt

Middle Atlas, $14 \mathrm{~km}$ from Azrou on road to Ain Leuh, 11 June [12]

10500 Pseudevernia furfuracea (L.) Zopf

Middle Atlas, $7 \mathrm{~km}$ from Azrou along road to Midelt, 12 June [14]

10502 Anaptychia ciliaris (L.) Koerb.

10504 Ramalina fraxinea (L.) Ach.

10506 Xanthoria parietina (L.) Th. Fr.

10507 Usnea

10508 Melanohalea exasperate (De Not.) O.Blanco \& al. 
10509 Lecidella elaeochroma (Ach.) Choisy

10510a Physcia aipolia (Humb.) Fuernr.

10510b Lecanora carpinia (L.) Vain.

Middle Atlas, $34 \mathrm{~km}$ from Azrou on road to Midelt, 12 June [17]

10520 Caloplaca schistidii Anzi

10521 Dermatocarpon

10523 Physconia perisidiosa (Erichsen) Moberg

C. $25 \mathrm{~km} \mathrm{~S}$ of Saka on road from Guercif to Nador, 14 June [21]

10533 Aspicilia

10534 Acarospora sulphurata (Arnold) Arnold

C. $55 \mathrm{~km}$ from Nador on road to Guercif, 14 June [22]

10539a Collema

10539b Gloeoheppia turgida (Ach.) Gyein.

10541 Fulgensia

10545 Squamarina lentigera (Web.) Poelt

C. $4 \mathrm{~km}$ from village of Ain Zorah on road from Saka, 14 June [23]

10549 Cladonia

10555a Fulgensia

10555b Squamarina

10556 Diploschistes

Middle Atlas, $18 \mathrm{~km}$ from Taza along minor road to Gouffre de Friouato and

Jbel Tazekka, 15 June [26]

10570 Parmelia pulla Ach.

10571 Lepraria

10574 Cladonia pocillum (Ach.) Grognot

Middle Atlas, $42 \mathrm{~km}$ from Taza on minor road, 15 June [27]

10576 Cladonia fimbriata (L.) Fr.

10577 Parmelia tiliacea (Hoffm.) Ach.

10578 Physconia pulverulacea Moberg

10579 Cladonia foliacea (Huds.) Willd.

10580 Anaptychia ciliaris (L.) Koerb.

10581 Lecidea

10582 Aspicilia

10583 Bacidia rubella (Hoffm.) A.Massal.

Middle Atlas, around summit of Jbel Tazekka, 16 June [29]

10591 Platismatia glauca (L.) Culb. \& Culb.

10592 Anaptychia ciliaris (L.) Koerb.

10593 Pertusaria

10594 Caloplaca

10596 Parmelia saxatilis (L.) Ach.

10597 Rhizocarpon 
10598a ?Aspicilia

10598b Parmelia pulla Ach.

10599 Rhizocarpon

Middle Atlas, c. 13 km SSW of Taza on minor rd to Bab-Bou-Idir, 16 June [33]

10605 Anaptychia ciliaris (L.) Koerb.

10606 Ramalina fraxinea (L.) Ach.

10607 Xanthoria parietina (L.) Th. Fr.

C. $37 \mathrm{~km}$ from Taza on rd to Nador, S of Dar Caïd-Medboh, 17 June [34]

10608 Lecanora crenulata (Dicks.) Hook.

10609a ?Aspicilia

10609b Diploschistes

Junction on Taza-Aknoul road with turning to Mesquitem, 17 June [38]

10616 Xanthoria parietina (L.) Th. Fr.

C. $15 \mathrm{~km} \mathrm{SW}$ of Ketama along road to Taounate and Fès, 19 June [43]

10631 Toninia

C. $4 \mathrm{~km}$ along track to Jbel Tidirhine, $9 \mathrm{~km}$ from Ketama, 20 June [44]

10641 Cladonia

10642 Ramalina farinacea (L.) Ach.

10643 Pseudevernia furfuracea (L.) Zopf

10644 Platismatia glauca (L.) Culb. \& Culb.

10645 Anaptychia ciliaris (L.) Koerb.

10646 Parmelia saxatilis (L.) Ach.

10647 Platismatia glauca (L.) Culb. \& Culb.

10648 Physcia leptalea (Ach.) DC.

10649 Physconia venusta (Ach.) Poelt

10650 Pertusaria

10651 Pertusaria

10652 Nephroma lusitanicum Schaerer

10653 Hypogymnia

10654 Caloplaca

10671 Cladonia fimbriata (L.) Fr.

C. $12 \mathrm{~km}$ along track to Jbel Tidirhine, 20 June [45]

10672 Letharia vulpina (L.) Hue

C. $14 \mathrm{~km}$ along track to Jbel Tidirhine, 20 June [46]

10683 Cladonia fimbriata (L.) Fr.

10684 Lecanora

10685a Aspicilia

10685b Rhizocarpon

C. $9 \mathrm{~km}$ along track to Jbel Tidirhine, 20 June [47]

10699 Perusaria albescens (Huds.) Choisy \& Werner

10700 Nephroma laevigatum Ach.

C. $23 \mathrm{~km}$ from Ouazzane on road to Chefchaouèn, 23 June [52] 
10742 Evernia prunastri (L.) Ach.

10744 Cladonia rangiformis Hoffm.

C. $5 \mathrm{~km}$ up track to Jbel Tizirane, $74 \mathrm{~km}$ from Chefchaouèn 24 June [57]

10813 Pseudevernia furfuracea (L.) Zopf

10820 Platismatia glauca (L.) Culb. \& Culb.

10822 Parmelia quercina (Willd.) Vain.

10823 Lobaria scrobiculata (Scop.) DC.

10825 Anaptychia ciliaris (L.) Koerb.

10826 Lobaria pulmonaria (L.) Hoffm. var. meridionalis (Vain.) Zahlbr.

10830 Degelia plumbea (Lightf.) Jøerg.\& James

10831 Ochrolechia pallescens (L.) A. Massal.

10832 Rhizocarpon geographicum (L.) DC.

C. $44 \mathrm{~km}$ from Chefchaouèn, $14 \mathrm{~km}$ above Talembote on track to Jbel Tassaout 25June [60]

10914 Evernia prunastri (L.) Ach.

10915 Perusaria amara (Ach.) Nyl.

C. $38 \mathrm{~km}$ from Chefchaouèn, $14 \mathrm{~km}$ above Bab Taza on track on Jbel

Talassemtane, by forest house, 26 June [64]

10951 Caloplaca ferruginea (Ach.) Branth

10954 Lecidella elaeochroma (Ach.) Choisy

10955 Pertusaria

10956 Squamarina cartilaginea (With.) P. James 